\title{
Development of Cabin and Plant Automation Prototype for Industrial Application
}

\author{
Ms. Sadhana Tiwari, Ms. Neha Sharma \\ ECE Assistant Professor, \\ Prestige Institute of Engineering, Management \& Research, \\ Indore (M.P)
}

\begin{abstract}
The Prototype for industrial automation application is designed to help in industrial work to monitor and work easily. This paper presents a low cost and flexible cabin control and weight measurement system. It is based on embedded micro web server in Arduino Mega 2560 microcontroller, with IP connectivity for accessing and controlling devices and appliances remotely. To demonstrate the feasibility and effectiveness of this system, devices such as light switches, power plug, temperature sensor, gas sensor, water flow meter, motion sensor and also control DC motor have been integrated in proposed prototype.
\end{abstract}

Keywords:-Arduino Uno, Arduino Mega, Sensors, Automation

\section{INTRODUCTION}

With the recent trends of technology, all systems or process are being automated. Automation is used to monitor and control various systems or machines to improve product quality and to reduce human efforts. Automation is known as Labor-saving technology. In Today's Scenario highly increasing competitiveness over the industry demands high quality and most consistent products with a competitive price. To accepts this challenges, Industrial automation is introduced which is used to increase the product quality, reliability and production rate while reducing production and design cost by adopting new, innovative and integrated technologies and services. This paper proposes the internet based cabin and plant automation system model focuses on monitor and controlling cabin and plants electronic devices whether you are inside or outside your Cabin and Plant automation gives an individual the ability to remotely or automatically control things around the Cabin and Plant. In Automation devices are being controlled automatically by turning ON/OFF certain devices and beyond, either remotely. A Cabin and Plant appliance is a device which is designed to perform in many electrical appliances, such as a refrigerator, household use.

In this paper we propose an embedded micro-web server with Arduino Mega 2560 microcontroller is introduces with IP connectivity. This Web server is used to access and control appliances through remotely. Proposed system does not

require any dedicated server. In addition to this, it offers some communication protocol through which we monitor and control cabin and plants environment remotely. Smart Cabin is equipped with many electronic devices that can be monitor and control remotely by internet.

\author{
Ms. Shelin Saiyyad*, Ms. Rebeca Kujur, \\ ECE Student, \\ Prestige Institute of Engineering, Management \& Research, \\ Indore (M.P)
}

\section{LITERATURE SURVEY}

The early life of automation in manufacturing industry has started with the use of basic hydraulic and pneumatic system. Automation is used to monitor and control various systems or machines to enhance product quality, production and to reduce human efforts. In recent industry demands high quality and most consistent products with a competitive price. To accept these challenges, Latest technology like Industrial automation is introduced through which all industrial operation is being automated with improved efficiency and production. In 1913 Ford Motor company comes with the idea of automation. Before then, a car was built by a team of skilled and unskilled workers. This Company introduced assemblyline which was automated. Through the concept of automated assembly line, it reduces the car assembly time from 12 hours per car to about one and a half hours per car and increase mass car production. Modern technology is transforming the world by generating opportunities that weren't available just a decade ago. In manufacturing, for example, IoT and connected devices are making the development process more transparent. The technology also allows plant managers and overseers to accurately review operations, which leads to a more positive direction for everyone.Most automated machinery and tools can operate at an optimal level, as well as improve working conditions for those spending the most time inside such facilities.

IOT (internet of things) is a technology that makes use of control systems such as computer to control the physical devices through the internet. Here we propose efficient industry automation system that allows user to work to efficiently control industry appliances/machines over the internet.

\section{SYSTEM OVERVIEW:}

A micro-controller is used to obtain values of physical conditions through sensors connected to it [These integrated sensors such as the temperature sensor read temperature values, the gas sensor detects smoke and fire outbreak. The automatic switching of the light is controlled by the Light Dependent Resistor (LDR) which determines the day light intensity. Also to incorporate security in our design, a motion detector is integrated using Passive Infrared Sensor (PIR) to detect movement in the home when the security system is turned on. 
The Process has been developed based on IOT (INTERNET OF THINGS). An interface card is introduced which provides the communication between the remote user, server, raspberry pi card and the home Appliances. This applicationis installed on an android Smartphone, a web server, and a raspberry pi card to control and monitoring the shutter of windows. Android application on a Smart phone issuescommand to raspberry pi card. An interface card is used to update signals between the actuator sensors and the raspberry pi card. In the present Scenario, security systems are crucial for protection of lives. Securitycan be achieved by the incorporation of various subsystems surveillance, intruder control, access control, fire detection, etc with single control unit. A smart Cabin is equipped with several devices that can be controlled remotely An internet based cabin and plant automation system focuses on controlling cabin and plants electronic devices whether you are inside or outside your Cabin and Plant. Using a WIFI shield to act as a Micro web server for the Arduino eliminates the need for wired connections between the Arduino board and computer which reduces cost and enables it to work as a standalone device. The Wi-Fi shield needs connection to the internet from a wireless router or wireless hotspot and this would act as the gateway for the Arduino to communicate with the internet.

Plant Automation:

Plants offer feature such as environmental monitoring using the temperature, humidity, water flow meter which is measure rate of liquid. It also offers switching functionalities to control motor speed,rpm, and other plants appliances connected to the motor driver system. Another feature of this system is the control the valve of the tank which it offers using the high level sensor and low level sensor and all these can be controlled from the website.

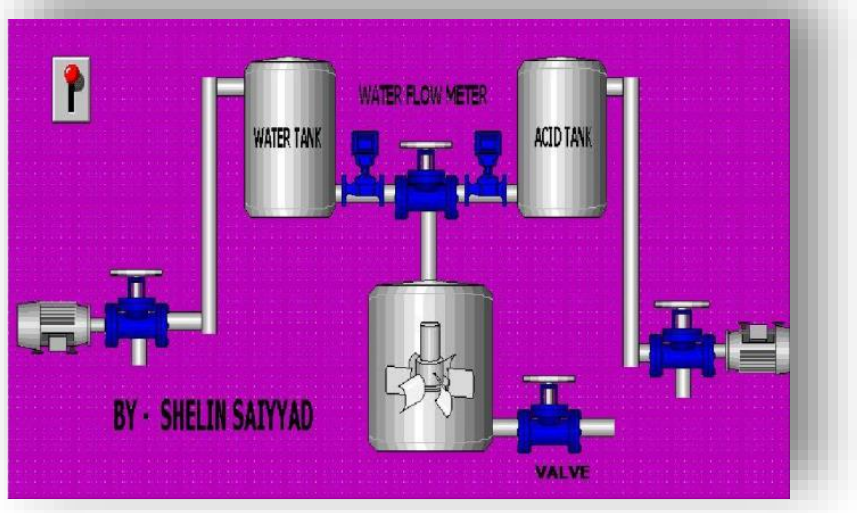

Fig1. Plant Automation Image

Cabin Automation:

Smart cabin offers feature such as environmental monitoring using the temperature, humidity, gas and smoke sensors. It provides many switching functionalities like to control lighting, fans/air conditioners, and other home appliances connected to the relay system. Another feature of this system is the intrusion detection which it offers using the motion sensor and all these can be controlled from the website.

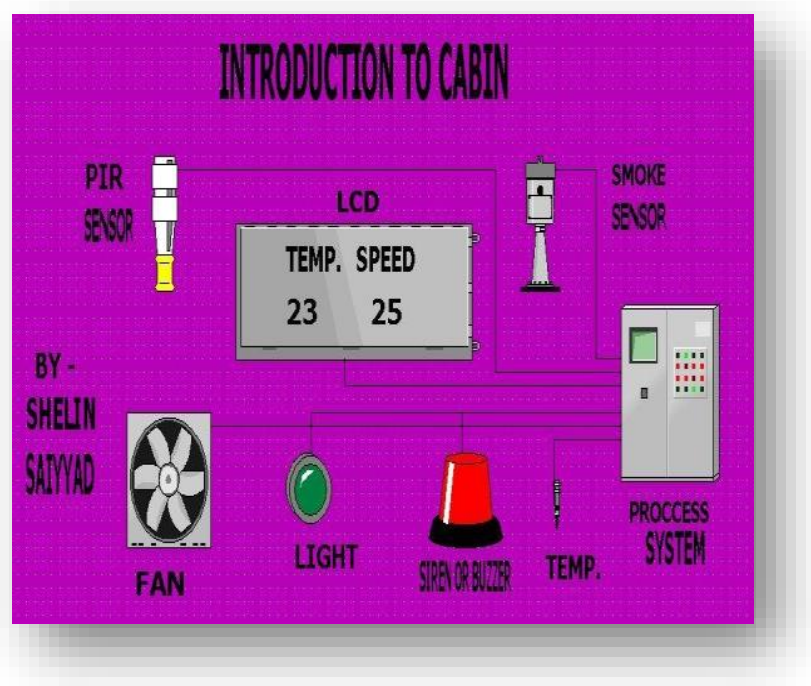

Fig2. Cabin Automation Image Drawn by SCADA Weight Machine Automation:-

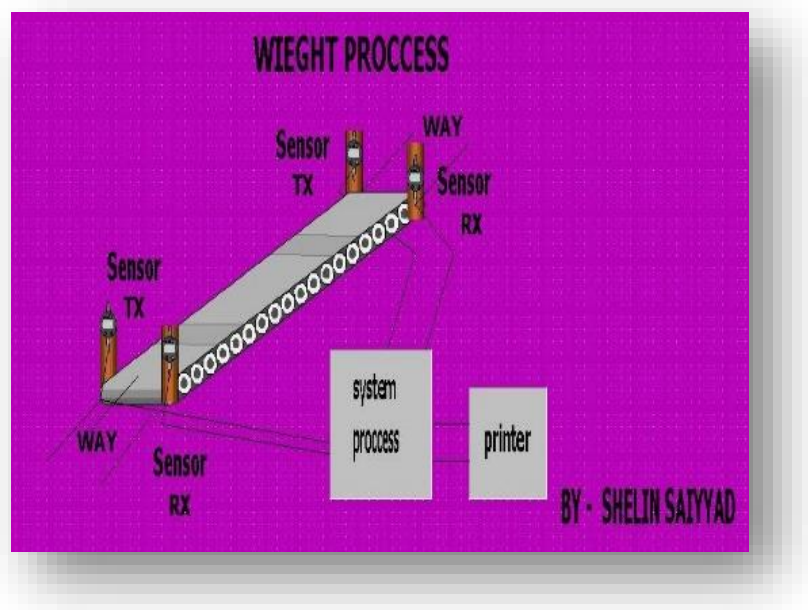

Weight machine is basically used for weight in which we will implement a security for accurate measurement. We will use two transmitters and two receivers. Transmitter will be send that causes a piston or turbine to rotate. The volumetric continuously signal to the receivers and receiver is received signal from the transmitter. Whenever any cut off between transmitter and receiver so that time weight machine will be not working. This process will be proper show on the website. We will monitor continuous monitor every moment on the website by using internet.

\section{SYSTEM COMPONENT}

1. Arduino mega

Arduino is an open source platform which is based on hardware and software.Arduino mega consist of 54 digital i/o pins and 16 analog pins in corporate on the board that 
make this device unique. Out of 54 digital i/o, 15 pins are used for pwm (pulse width modulation).

2. DHT 11 sensor

The dht11 is a ultra-low-cost digital temperature and humidity sensor. It measures the surrounding air, and give digital signal on the data pin and there are no need analog input pins needed. For humidity sensing it uses two electrodes with moisture holding substrate between them.

3. LCD

It stands for liquid crystal display. It is a flat panel display technology which is used in many electronics devices like tv, smart phones, laptop and many more. It is available in different sizes like $16 \times 2$ characters, $16 \times 4$ characters, $20 \times 4$ characters and many more.

4. Smoke Detector:

Smoke detector is also known as smoke alarms. It is a self-contained safety device that can be placed anywhere to detect smoke and sounding an alarm to alert occupants.

\section{Waterflow meter}

It measures the speed of water flowing through the pipe that causes a piston or turbine to rotate. The volumetric flow rate of the water is proportional to the rotational speed of the blades.

6. Dc motor

Dc motor converts electrical energy into mechanical energy. In dc motor is assembled with multiple gear setups. Speed of motor is counted in terms of rotations of the soft per minute is called revolution per/ minute. The setup assembles help to increasing the torque and reduces the motor speed. This type of dc motor can be used in all micro-controller based robots.

7. LDR sensor

LDR or light dependent resistor is also known as photo resistor, photocell, and photo-conductor. $\mathrm{Ldr}$ is a resistor whose resistance depend on the amount of light falling on its surface. When the light incident on surface, then it resistance changes. A typical light dependent resistor has a resistance in the darkness of $1 \mathrm{mohm}$, and in the brightness a resistance of a couple of kohm.

\section{Fire detector}

Fire detector is also known as smoke detector but it also detectsheat, light, gas, infrared and ultraviolent light radiations.It enables a person which physically detects fire to raise the alarm,flashers, and control panel which is the brain ofthe system.

\section{RESULTS:}

The Prototype for industrial automation was designed in which two features are added first cabin automation and second one is plant automation using Arduino board for programming and interfacing purpose. This prototype is capable for measuring and monitoring the temperature, flow rate of liquid and speed of the motor.Display images are shown below:
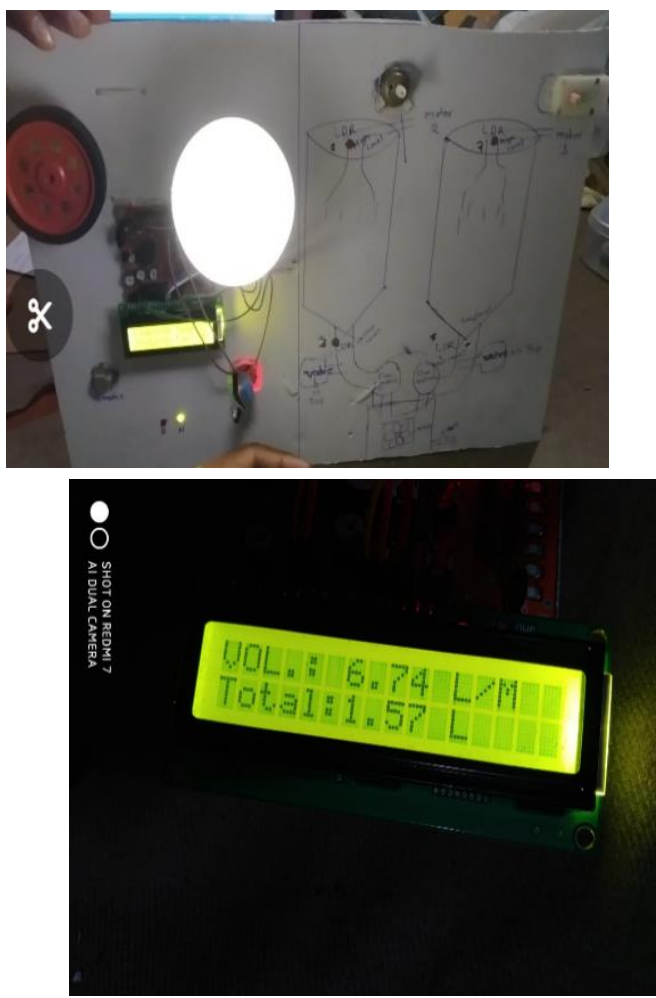

CONCLUSION:

In this paper, automatic plant and cabin automation model is proposed, developed and tested in laboratory using an embedded micro-web server with Arduino Mega and various sensor devices. Proposed model can be used in offices, cabin and plants. Automatic weight measurement is also proposed and tested under laboratory condition. This method is cheaper, accurate and easy to use. With the help of this prototype various parameters are continuously monitored automatically and these models are feasible to implement in real word scenario.

\section{REFERENCES:}

[1] BohnH,BobekA,GolatowskiF infrastructurefor

(2006)Sirena-service timeembeddednetworkeddevices:Aserviceorientedframeworkfordif fer-ent domains.In Networking, InternationalConferenceonSystemsand InternationalConferenceonMobileCommunicationsandLearningTechnol ogies,2006.ICN/ICONS/MCL2006.InternationalConferenceon,pp4 3-43,DOI10.1109/ICNICONSMCL.2006.196

[2] BonyB, Harnischfeger M, JammesF(2011) Convergenceofopcuaanddpws with a cross-domain data model. In: Industrial Informatics $\quad$ (INDIN), 2011 9thIEEEInternationalConferenceon, $\quad$ pp187192,DOI10.1109/INDIN.2011.6034860

[3] BoydA, NollerD, PetersP, Salkeld D, ThomasmaT, Gifford C,PikeS,Smith A(2008)SOAinmanufacturingguidebook.Tech.rep.,IBMCorporation,MESAInternationaland Capgemini,URL-

ftp://public.dhe.ibm.com/software/plm/pdif/MESA_SOAinManufa cturingGuidebook.pdf

[4] Candido G, Jammes F, Barata J, Colombo A (2009) Generic management ser-vicesfordpws-enableddevices. 
In:IndustrialElectronics,2009.IECON'09.35th Annual Conference ofIEEE,pp 3931-3936, DOI 10.1109/IECON.2009.5415339

[5] Chowanetz M, Pfarr F, Winkelmann A (2013) Amodel of critical success fac-tors forsoftware-as-a-service adoption.In: 7th IFAC Conference onManufac-turing Modelling, Management, and Control, St. Petersburg, Russia

[6] ChowdhuryMW,IqbalMZ(2004)Integrationoflegacysystemsinsoft warearchitecture.In:SpecificationandVerificationofComponentBasedSystems(SAVCBS) Workshop at ACM SIGSOFT 2004/FSE-12

[7] Colombo A, Mendes J, LeitaoP, Karnouskos S (2012) Serviceoriented scadaandmessupportingpetrinetsbasedorchestratedautomationsyste ms. In: IECON201238thAnnualConferenceonIEEEIndustrialElectronicsSo-ciety, pp 6144-6150, DOI 10.1109/IECON.2012.6389076

[8] ColomboAW,KarnouskosS(2009)Towardsthefactoryofthefuture:A service-oriented cross-layer infrastructure.In: ICT Shapingthe World:A Sci-entificView,vol6581,EuropeanTelecommunicationsStandardsInstitute(ETSI), John Wiley and Sons

[9] ColomboAW,KarnouskosS,MendesJM(2010)Factoryofthefuture:A service-oriented system of modular, dynamic reconfigurable and collaborativesystems.In:BenyoucefL, GrabotB(eds)ArtificialIntellig enceTechniques24This is a preprint version, which may deviate from the final version which can be acquired from Bangemann et al Reference forNetworkedManufacturingEnterprisesManagement,ISBN978-184996-118-9, Springer

[10] ComellaDordaS,WallnauK,SeacordR,RobertJ(2000)Asurveyofblac $\mathrm{k}$ boxmodernizationapproachesforinformationsystems.In:Proceeding softheInternationalConferenceonSoftwareMaintenance(ICSM'00),I EEEComputerSociety,Washington,DC,USA,ICSM'00,pp173,URLhttp://dl.acm.org/citation.cfm?id=850948.853443

[11] https://epicindustrialautomation.com/blog/automationimplementation-process

[12] https://www.manufacturingtomorrow.com/article/2018/08/tipsand-tricks-for-implementing-automation-in-your-manufacturingplant $/ 12042$ 